\title{
Numerical simulation of unsteady viscous flow around a circular cylinder
}

\author{
P MANIKYALA RAO* and K KUWAHARA \\ The Institute of Space and Astronautical Science, Sagamihara, Kanagawa \\ 229, Japan \\ * Present Address: Prof M Kawahara Lab, Civil Engineering Department, \\ Chuo University, Kasuga, Bunkyo-Ku, Tokyo 112, Japan
}

MS received 21 February 1990; revised 20 September 1990

\begin{abstract}
A finite difference solution is obtained for the time-dependent viscous incompressible 2-dimensional flow past a circular cylinder by direct integration of the Navier-Stokes equations expressed in a general curvilinear coordinate system. The solution describes the development of the vortex street developed behind the cylinder. Evolution of flow configuration is studied by means of streamlines, pressure contours, and vorticity contours for different Reynolds numbers. The time-dependent lift and drag coefficients are also obtained.
\end{abstract}

Keywords. Marker and cell method; vortex shedding; Strouhal number; finite difference method.

\section{Introduction}

One of the classical problems in fluid mechanics is the time-dependent viscous flow induced by an impulsively started circular cylinder. Despite the simplicity of the body geometry, the flow structure is complex and all the phenomena of fluid mechanics such as separation, transition, wake instabilities and fluid--structure interactions are present. A great deal of theoretical, experimental and numerical research has been devoted to the understanding of the near and far wake of bluff bodies, in general, and of a circular cylinder in particular. Numerical solutions play an important role in such investigations since they can, in principle, be carried out for any value of the Reynolds number. Many theoretical and numerical investigations have been reported in the literature (Thom 1933; Kawaguti 1953; Kawaguti \& Jain 1966; Takami \& Keller 1969; Collins \& Dennis 1973; Patel 1976; Kuwahara 1978; Sarpkaya \& Schoaff 1979; Bouard \& Coutanceau 1980; Phuoc Loc \& Bouard 1985). Besides these theoretical and numerical investigations, some experimental visulizations have been described by Honji \& Taneda (1969), Taneda (1977), Coutanceau \& Bouard (1977), Gerrard $(1967,1978)$ and Wei \& Smith (1986).

High Reynolds number flow around a circular cylinder has so far been studied almost exclusively by experiments. However recent development of high speed 
computers and the numerical methods have enabled us to compute the flow with separation at quite high Reynolds numbers by direct integration of the Navier-Stokes equations using the finite-difference method (Kawamura \& Kuwahara 1984, 1985; Manikyala Rao \& Kawahara 1990; Manikyala Rao et al 1990, and unpublished).

The main purpose of this paper is to analyse the flow structure of the 2-dimensional impulsively started circular cylinder at high Reynolds numbers. The vortex street development behind the cylinder is obtained. Evolution of flow structure is studied by means of streamlines, pressure contours and vorticity contours for Reynolds numbers, $\mathrm{Re}=1000,5000,9500$ and 40000 , and the time-dependent lift and drag coefficients obtained. As the computations are performed only in a two-dimensional configuration, the secondary instabilities developed in the wake may not be exactly the same as those occurring in a physical experiment. Hence, the analysis carried out here is only a rough, qualitative comparison. However, these results could be used in comparison with a physical experiment, or with other three-dimensional numerical results, in order to understand how the turbulence appearing after separation and three-dimensional motion affect the evolution of the different structures in the wake, and also examine whether there are some features of the near wake structures that remain two-dimensional.

\section{The mathematical modelling}

The equations governing the unsteady flow of an incompressible viscous fluid past a circular cylinder are the continuity and Navier-Stokes equations.

In terms of the dimensionless variables,

$$
x^{*}=x / d, y^{*}=y / d, u^{*}=u / U, v^{*}=v / U, t^{*}=t(U / d), p^{*}=p /\left(\rho U^{2}\right),
$$

the flow is governed by the following equations

$$
\begin{aligned}
& \frac{\partial u}{\partial x}+\frac{\partial v}{\partial y}=0 \\
& \frac{\partial u}{\partial t}+u \frac{\partial u}{\partial x}+v \frac{\partial u}{\partial y}=-\frac{\partial p}{\partial x}+\frac{1}{\operatorname{Re}}\left(\frac{\partial^{2} u}{\partial x^{2}}+\frac{\partial^{2} u}{\partial y^{2}}\right) \\
& \frac{\partial v}{\partial t}+u \frac{\partial v}{\partial x}+v \frac{\partial v}{\partial y}=-\frac{\partial p}{\partial x}+\frac{1}{\operatorname{Re}}\left(\frac{\partial^{2} v}{\partial x^{2}}+\frac{\partial^{2} y}{\partial y^{2}}\right)
\end{aligned}
$$

where $p$ is the pressure, $\rho$ is the density, Re denotes the Reynolds number based on the velocity of the uniform flow, $U$, and the diameter, $d$, of the circular cylinder; $\operatorname{Re}=U d / v$ ( $v$ is the kinematic viscosity).

In the present study, the following coordinate transformation is introduced in order to simulate high Reynolds number flow precisely by taking fine meshes in the boundary layer and wake because the flow there is very complicated

$$
\xi=\xi(x, y), \quad \eta=\eta(x, y),
$$

where $(x, y)$ are the variables in the physical domain and $(\xi, \eta)$ are those of the computational domain. The inverse transformation of (4) is given by

$$
x=x(\xi, \eta), \quad y=y(\xi, \eta) .
$$


The basic equations in the transformed plane are written as

where

$$
\begin{aligned}
& J u_{t}+\left(u y_{\eta}-v x_{\eta}\right) u_{\xi}+\left(v x_{\xi}-u y_{\xi}\right) u_{\eta}=-\left(y_{\eta} p_{\xi}-y_{\xi} p_{\eta}\right)+J \operatorname{Re}^{-1} \nabla^{2} u, \\
& J v_{t}+\left(u y_{\eta}-v x_{\eta}\right) v_{\xi}+\left(v x_{\xi}-u y_{\xi}\right) v_{\eta}=\left(x_{\eta} p_{\xi}-x_{\xi} p_{\eta}\right)+J \operatorname{Re}^{-1} \nabla^{2} v,
\end{aligned}
$$

$$
\begin{aligned}
\nabla^{2} f= & \left(\alpha f_{\xi \xi}-2 \beta f_{\xi \eta}+\gamma f_{\eta \eta}\right) / J^{2}+\left[\left(\alpha x_{\xi \xi} 2 \beta x_{\xi \eta}+\gamma x_{\eta \eta}\right)+\left(y_{\xi} f_{\eta}-y_{\eta} f_{\xi}\right)+\right. \\
& \left.\quad+\left(\alpha x_{\xi \xi}-2 \beta x_{\xi \eta}+\gamma x_{\eta \eta}\right)\left(x_{\eta} f_{\xi}-x_{\xi} f_{\eta}\right)\right] / J^{3} \\
J= & \left(x_{\xi} y_{\eta}-x_{\eta} y_{\xi}\right) \\
\alpha= & \left(x_{\eta}^{2}+y_{\eta}^{2}\right) \\
\beta= & \left(x_{\xi} x_{\eta}+y_{\xi} y_{\eta}\right) \\
\gamma= & \left(x_{\xi}^{2}+y_{\xi}^{2}\right) .
\end{aligned}
$$

\section{Numerical analysis}

The numerical technique adopted in this paper is based on the well:-known marker and cell (MAC) method, which was originally developed by Harlow \& Welch (1965). In order to overcome numerical instability that may arise in high-Reynolds number computations, the third-order upwind scheme for the convection term is adopted (for more details, see Kawamura \& Kuwahara 1984).

First, the Poisson equation for the pressure is derived by taking the divergence of (3)

$$
\begin{aligned}
\nabla^{2} p & =\left(p_{x x}+p_{y y}\right)=-\operatorname{div}(\mathbf{V} \cdot \operatorname{grad}) \mathbf{V}+R, \\
R & =-(\partial / \partial t) D+\operatorname{Re}^{-1} \nabla^{2} D, \\
D & =\operatorname{div} \mathbf{V},
\end{aligned}
$$

where $\mathbf{V}=(u, v)$ and subscripts denote the partial derivatives.

If $\mathbf{V}$ is given at a particular time, then the Poisson equation for pressure is solved numerically to get $p$ and by substitution of these values into (3a) and (3b). $V$, one time step later, is calculated from $\partial \mathrm{V} / \partial t$ in $(3 \mathrm{a}, \mathrm{b})$. Although $R$ in $(8 \mathrm{a})$ is identically zero due to (2), a part of $R$ is retained here as a corrective term in order to prevent the accumulation of numerical errors. This is because in numerical computations the velocity computed using the pressure obtained by solving (7) (where $R=0$ ) does not always satisfy $D=0$, although $D=0$ implies $R=0$. If the right hand side of (8a) is discretized by the implicit Euler scheme one obtains

$$
R=-\left(D^{*}-D\right) / \Delta t+\operatorname{Re}^{-1} \nabla^{2} D^{*},
$$

where $D^{*}$ is $D$ at the next time step. By imposing $D^{*}=0$ in (8c), the value of $R$ is obtained as $R=D / \Delta t$. Equation (7) can be represented as

$$
\nabla^{2} p=-\operatorname{div}(\mathbf{V} \cdot \operatorname{grad}) \mathbf{V}+D / \Delta t
$$

The solutions obtained by solving $(3 \mathrm{a}, \mathrm{b})$ and $(8 \mathrm{~d})$ satisfy $D^{*}=0$ at any time. The basic pressure equation in the physical plane is given by

$$
p_{x x}+p_{y y}=-\left(u_{x}^{2}+2 u_{y} v_{x}+v_{y}^{2}\right)+\left(u_{x}+u_{y}\right) / \Delta t .
$$


The first term of the right hand side of (9) is derived from $-\operatorname{div}(\mathbf{V} \cdot \operatorname{grad}) \mathbf{V}$ by using the continuity equation. The corresponding equation in the transformed plane is

$$
\begin{aligned}
\tilde{\nabla} p=[ & \left.\left(y_{\eta} u_{\xi}-y_{\xi} u_{\eta}\right)^{2}+2\left(x_{\xi} u_{\eta}-x_{\eta} u_{\xi}\right)\left(y_{\eta} v_{\xi}-y_{\xi} v_{\eta}\right)+\left(x_{\xi} v_{\eta}-x_{\eta} v_{\xi}\right)^{2}\right] / J^{2}+ \\
& +\left(y_{\eta} u_{\xi}-y_{\xi} u_{\eta}+x_{\xi} v_{\eta}-x_{\eta} v_{\xi}\right) /(J \Delta t) .
\end{aligned}
$$

Secondly, the velocity at the next time step is obtained by solving the Navier-Stokes equation

$$
\left(\mathbf{V}^{n+1}-\mathbf{V}^{n}\right) / \Delta t+\left(\mathbf{V}^{n} \cdot \operatorname{grad}\right) \mathbf{V}^{n}=-\operatorname{grad} p+\operatorname{Re}^{-1} \nabla^{2} \mathbf{V}^{n}
$$

All the spatial derivatives except nonlinear terms in $(6 a, b)$ are approximated by second-order central difference schemes. The nonlinear terms are approximated by the modified second-order upwind scheme where the third-order error term is eliminated (for more details, see Kawamura \& Kuwahara 1984) as

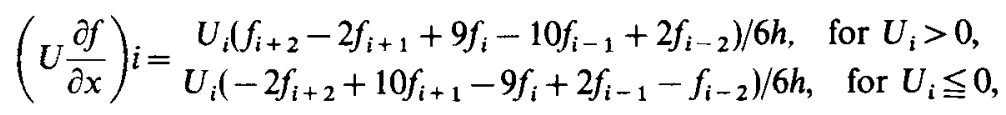

where $h$ is the grid spacing. The Poisson equation for the pressure is solved iteratively by using a modified successive over-relaxation (SOR) method. For the Navier-Stokes equations, the semi-implicit scheme which is equivalent to the Euler backward scheme except for the nonlinear convection term is used to integrate temporally. The convection term is linearized as

$$
V \cdot \operatorname{grad} V=V^{n+1} \cdot \operatorname{grad} V^{n+1} \sim V^{n} \cdot \operatorname{grad} V^{n+1}
$$

An O-grid system is chosen in this paper because the meshes near the body can more easily be controlled and are orthogonal to the body and the starting flow is convenient to compute. The mesh system and evaluation of $p, u$ and $v$ at the mesh points are shown in figure $1(a, b)$.

\section{Boundary and initial conditions}

The computational domain is enclosed by the body boundary, the inflow and downstream boundaries at a distance, and upper and lower boundaries, on which proper treatments are necessary (figure 2). The number of grid points are $200 \times 100$. The downstream outer boundary is located at a distance of $20 \mathrm{~d}$ from the centre of the cylinder. The time step is taken as 0.01 .

The boundary and initial conditions of the physical problem are

(i) no-slip condition on the surface of the cylinder $(u=v=0$ ),

(ii) uniform flow at infinity $(u=U, v=0)$,

(iii) the cylinder at rest initially in the fluid ( $u=v=0$ at $t=0)$.

Using the condition (i) in $6(a, b)$, the pressure can be obtained by extrapolation.

A periodic condition is used on the upper and lower horizontal boundaries, since the minimum number of grid points is used in the direction parallel to the axis of the cylinder. 
(a)

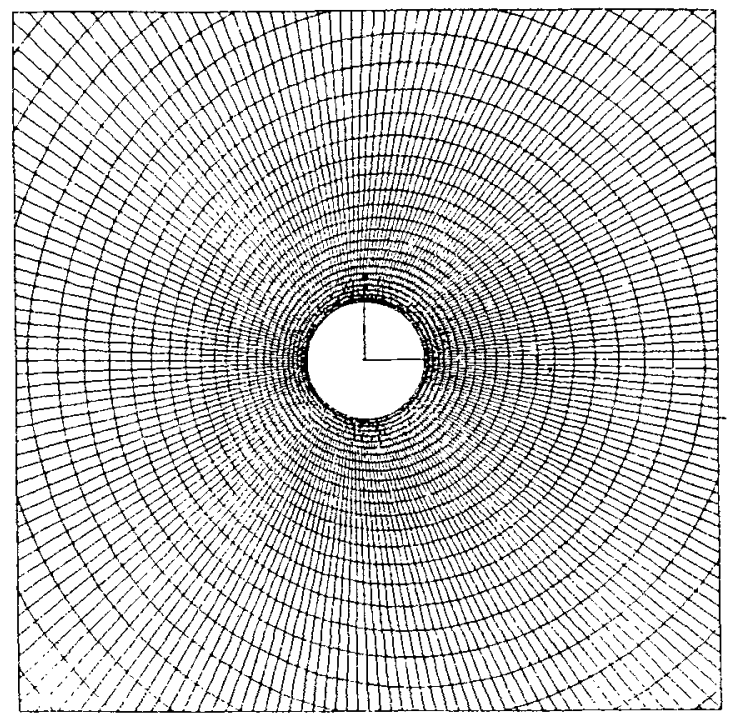

(b)

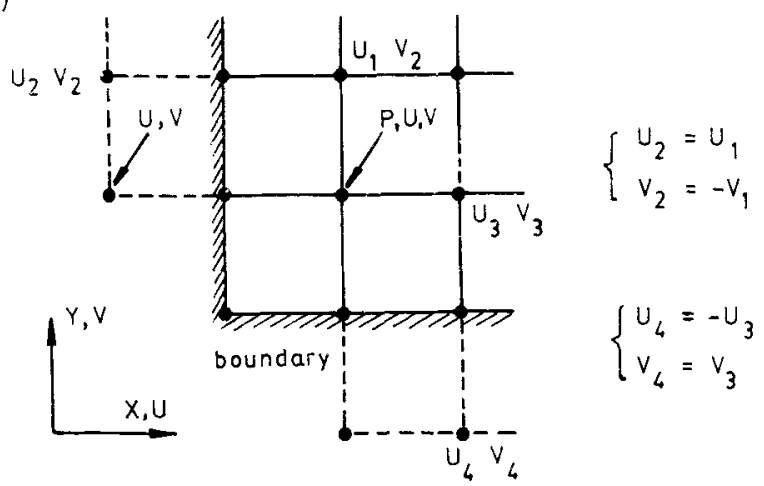

Figure 1. (a) Mesh system used in the computation, (b) placement of value of field variable about computational cell.

From the Courant condition for stability the following limitation on the time increment is derived

$$
\Delta t \leqq \Delta x /(\operatorname{Re} \cdot u)
$$

here $u$ is the radial velocity and $\Delta x$ is the smallest radial spacing (0.01).

\section{Discussion}

For the present study, the entire computation was carried out on a Fujitsu FACOM VP200 Super Computer. Figure 3 represents the development of the flow pattern computed for Reynolds number $\operatorname{Re}=1000$ at time $t=100$. The development of the vortex shedding is clearly seen. Two secondary vortices are formed, followed by a smaller single downstream of the separation point. As the vortices grow symmetrically, the shear layer joining the separation point to one of the vortices begins to develop instabilities and is drawn across the wake in response to the base pressure reduced by the action of the vortex growing across the wake. The stretching, diffusion, and 

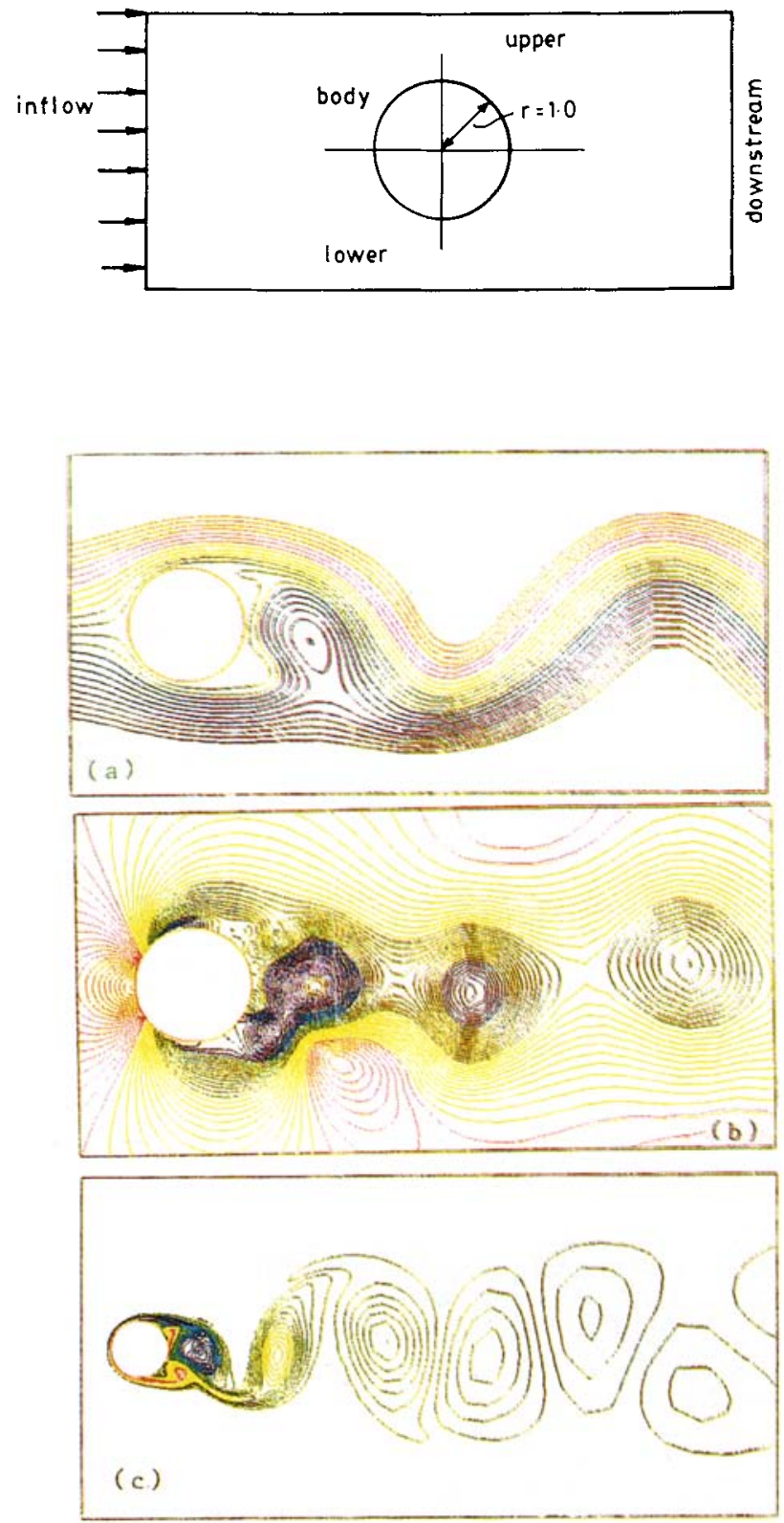

(d)

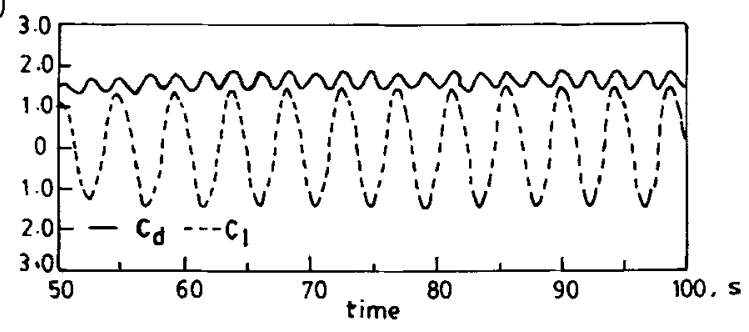

Figure 2. Schematic representation for the computational domain around a circular cylinder.
Figure 3. Instantaneous (a) streamline, (b) pressure contours. (c) vorticity contours, (d) time-dependent drag and lift coefficients for a circular cylinder for $R e=1000$, at time $=100$. 
dissipation of vorticity break up the deforming turbulent sheet and thereby the further supply of circulation to the vortex, whose rate of growth has already been reduced to its minimum. The vortex across the wake still continues to grow and entrains part of the oppositely signed vorticity. The shedding process for the second vortex does not commence until the circulation in its feeding sheet decreases to its minimum, making the sheet most susceptible to rapid diffusion. The eddies that develop in the near wake during a given period undergo successive interactions among themselves (figure $3 \mathrm{c}$ ). This configuration agrees with the experimental and numerical results. The unsteady lift coefficient oscillates periodically.

The pressure variations also illustrate the different categories of eddies and they correspond to the vortex-shedding phenomenon. The pressure contours also show engulfment of fluid from regions outside the wake (figure $3 \mathrm{~b}$ ). The corresponding streamline pattern is given in figure 3 a. However, one can notice that the secondary eddies at $\mathrm{Re}=1000$ are different from those appearing at higher Reynolds numbers at the end of the separated mixing layer (figure 4). The eddies of the separated mixing layer are more responsible for the laminar to turbulent transition than the secondary eddies for $\operatorname{Re}=1000$. The development of the flow pattern at time $t=100$, for $\mathrm{Re}=5000$ is presented in figure 4 .

The development of flow pattern for $\operatorname{Re}=9500$ at different times is shown in figure $5 \mathrm{a}$. The unsteady wake length behind the cylinder, measured by Phuoc Loc \& Bouard (1985), is compared with the present numerical results in figure $5 \mathrm{~b}$. The periodic properties of the flow are clearly shown by the time dependent evolution of the lift coefficient shown in figures $3 \mathrm{~d}$ and $4 \mathrm{~d}$. However, at $\mathrm{Re}=40000$ ripples are seen on the drag and lift curves (figure 6d), which indicate the periodical separation of small eddies from the surface, while at $\mathrm{Re}=1000,5000$ the ripples are very weak.

Figure 6 represents the development of the flow pattern for $\mathrm{Re}=40000$ at time $t=45$. Comparison of figures 6,3 and 4 shows that for higher Reynolds numbers the vortex street becomes irregular (Naumann et al 1966) and the streamwise interval of the vortices becomes large. Accordingly the value of lift changes temporally and becomes complicated with strong disturbances and is different from the simple sinusoidal curve (figure $6 \mathrm{~d}$ ).

Figure 7a shows the dependence of the mean drag coefficient on the Reynolds number, compared with experimental results (Schlichting 1968). Thi absolute values of the calculated drag coefficient are larger than the experimental data in all the computations. This is probably because the experimental conditions are different from the computational conditions. In the actual experiments the situation is threedimensional. Even uniform flow about a stationary cylinder exhibits chord-wise and span-wise variations. These three-dimensional effects may play a major role in the stretching of vortex filaments and in the redistribution of vorticity in all directions. The present computations are not in a position to account for such complex effects. The computations are performed in a two-dimensional configuration only. However, one may hope to assess the effects of three-dimensionality by means of twodimensional numerical experiments. Finally, figure $7 \mathrm{~b}$ shows the dependence of the Strouhal number on the Reynolds number compared with the experimental result of Roshko (1961). The computational results indicate that the turbulence phenomena of the fluid field including the separation region can be captured by the direct finite difference technique. 

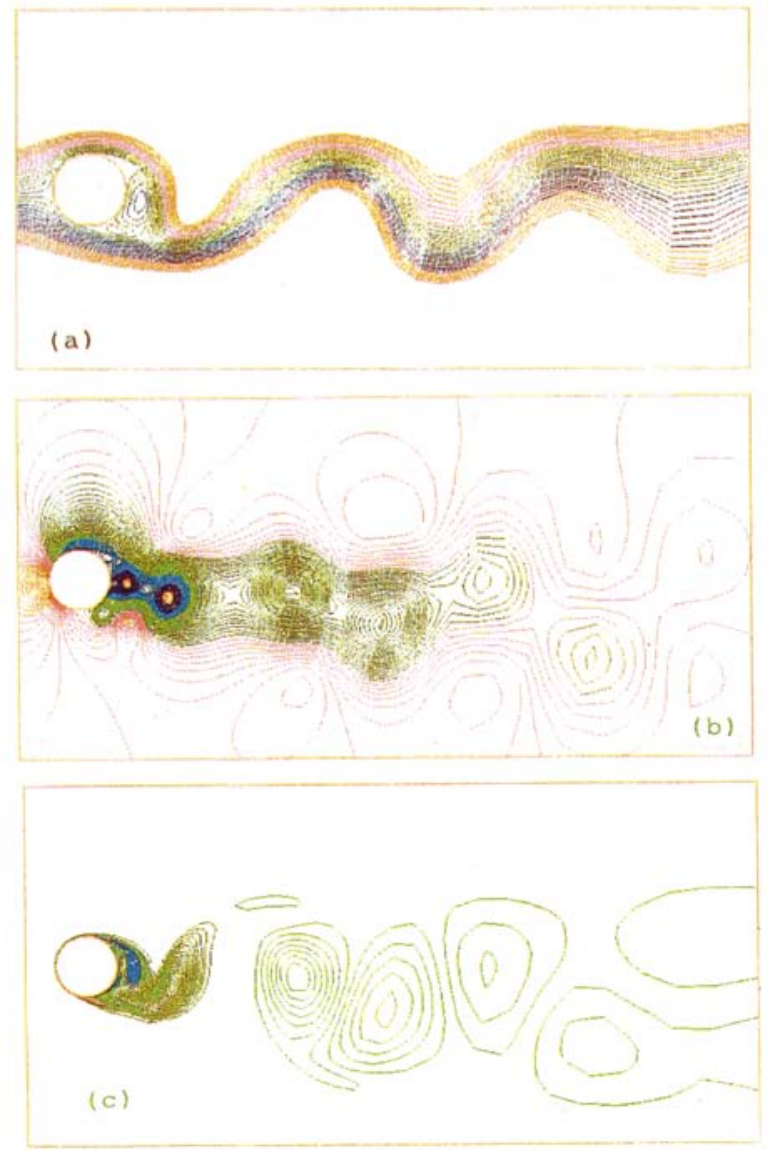

(d)

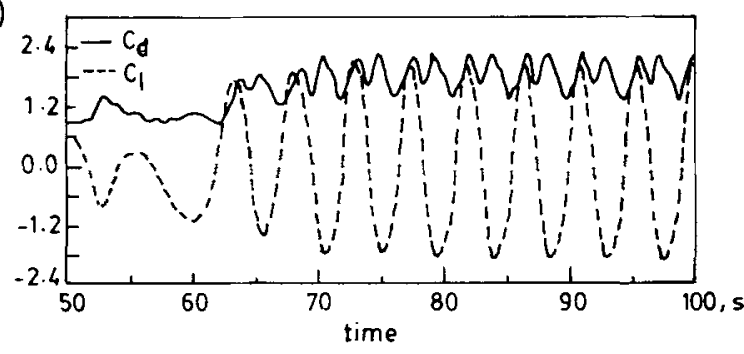

Figure 4. Instantaneous (a) streamlines, (b) pressure contours, (c) vorticity contours, (d) time-dependent drag and lift coefficients for a circular cylinder for $\operatorname{Re}=5000$, at time $=100$. 

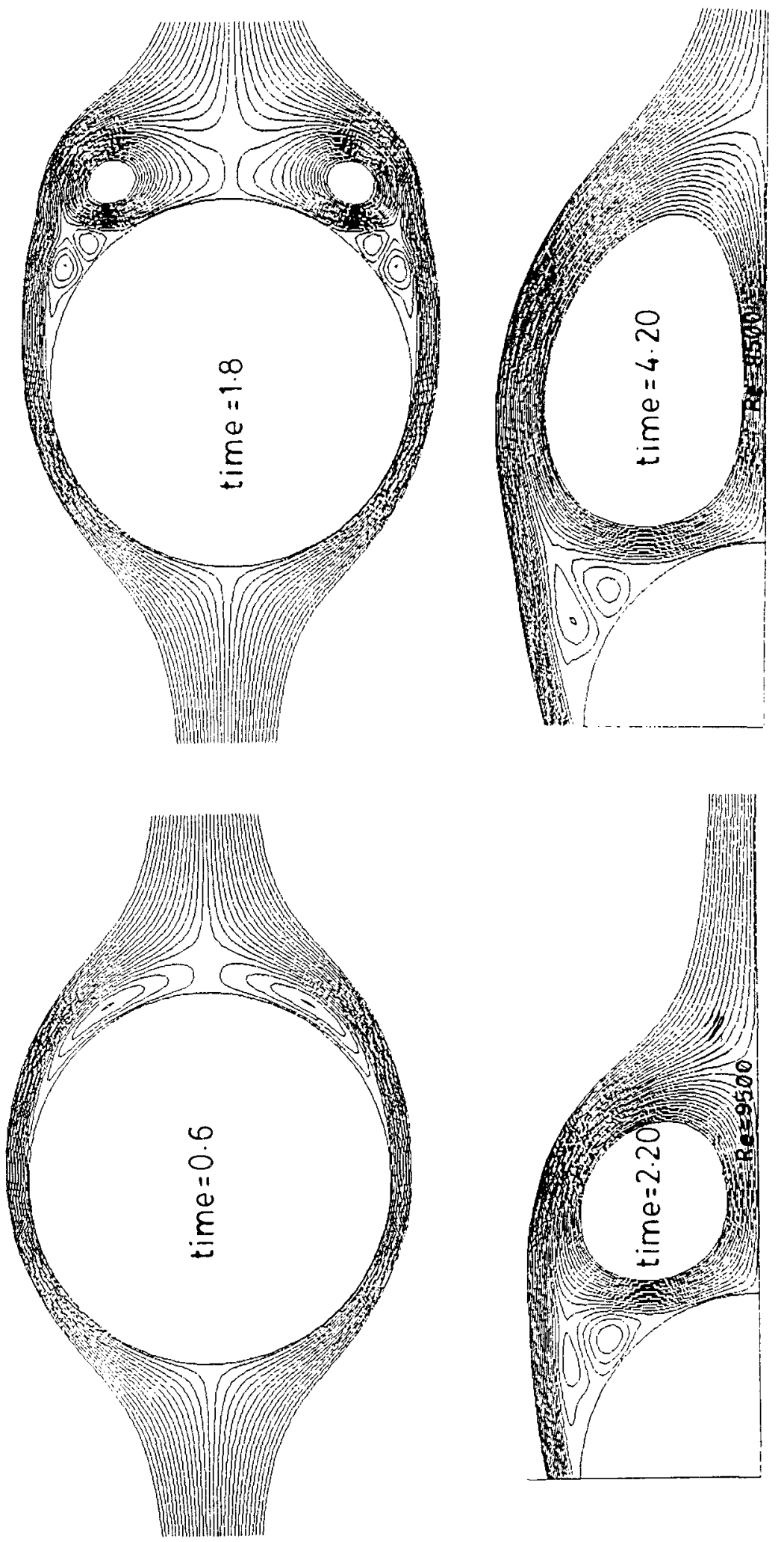

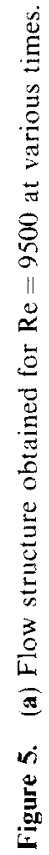



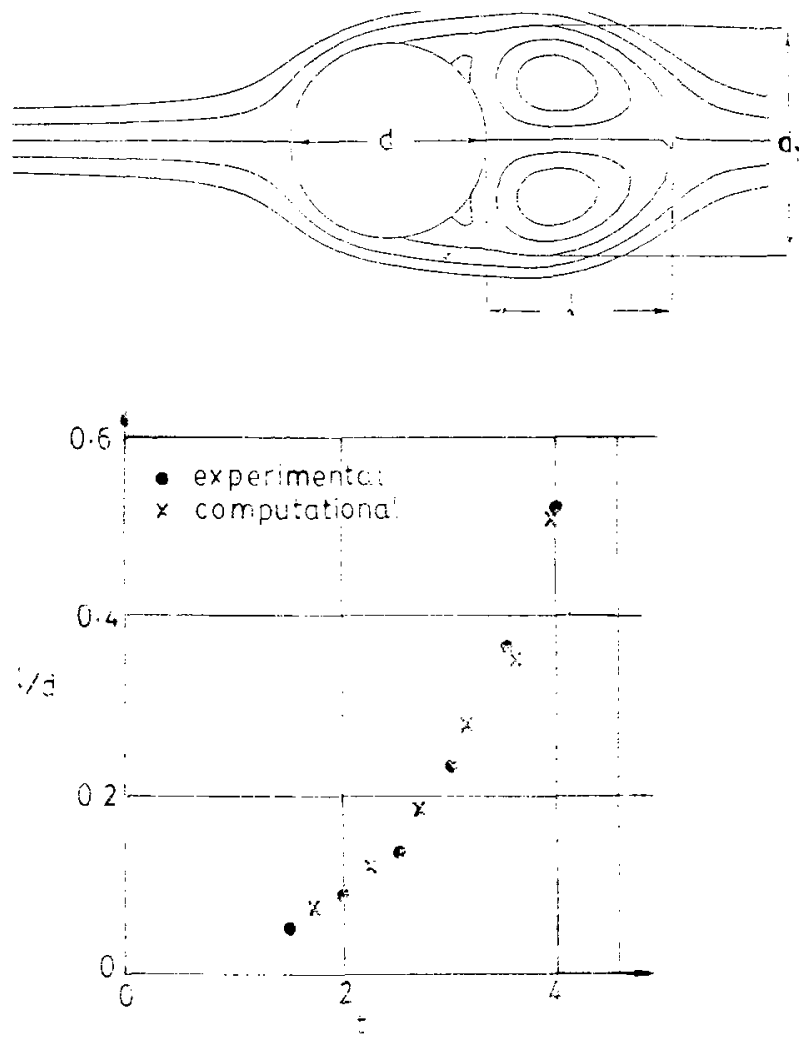

Figure 5. (b) Schematic siagram for the vortex iengtn. Comparsict between expenmental and numerica: results on the evolution with timis of the main vortex lengt:
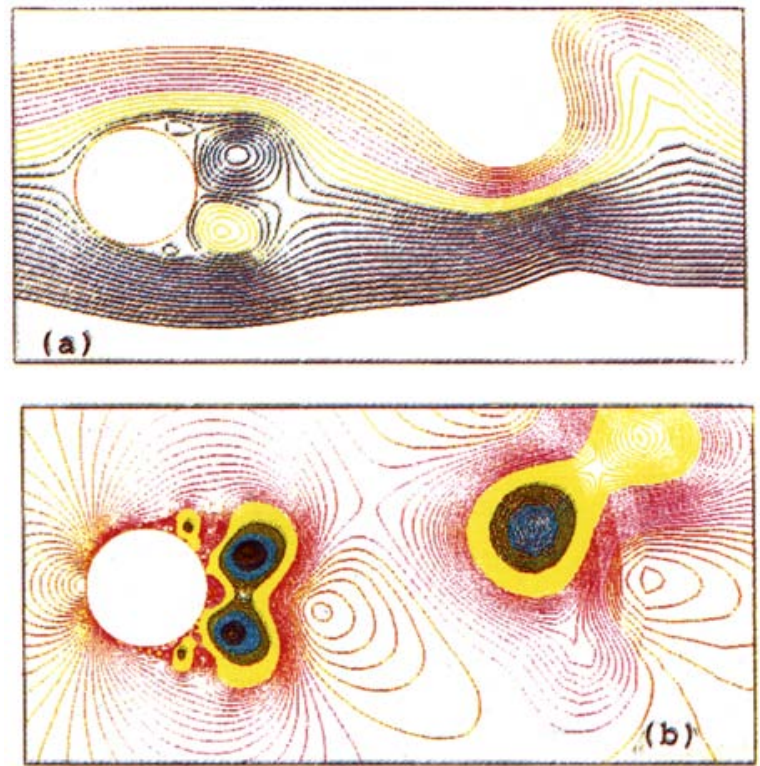

Figure 6 Instantaneous (a) streamlines. (b) pressure contours for a circular cylinder for $\mathrm{Re}=40,000 \mathrm{a}$ : time $=45$. 


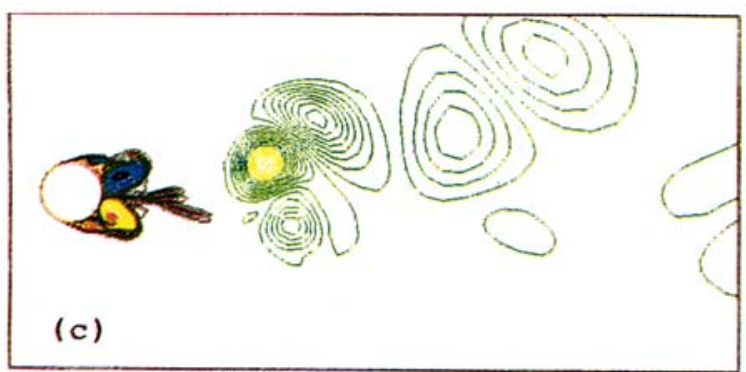

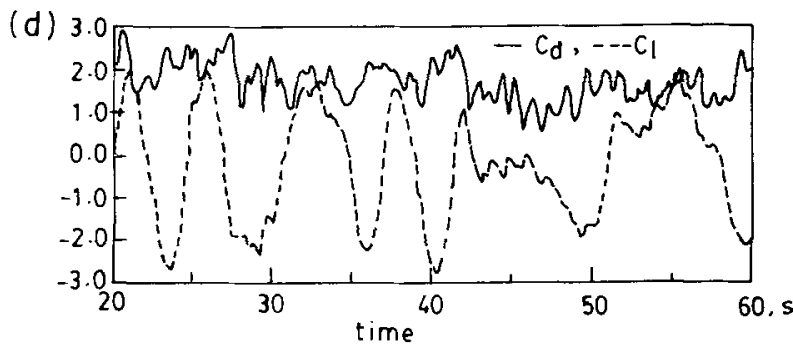
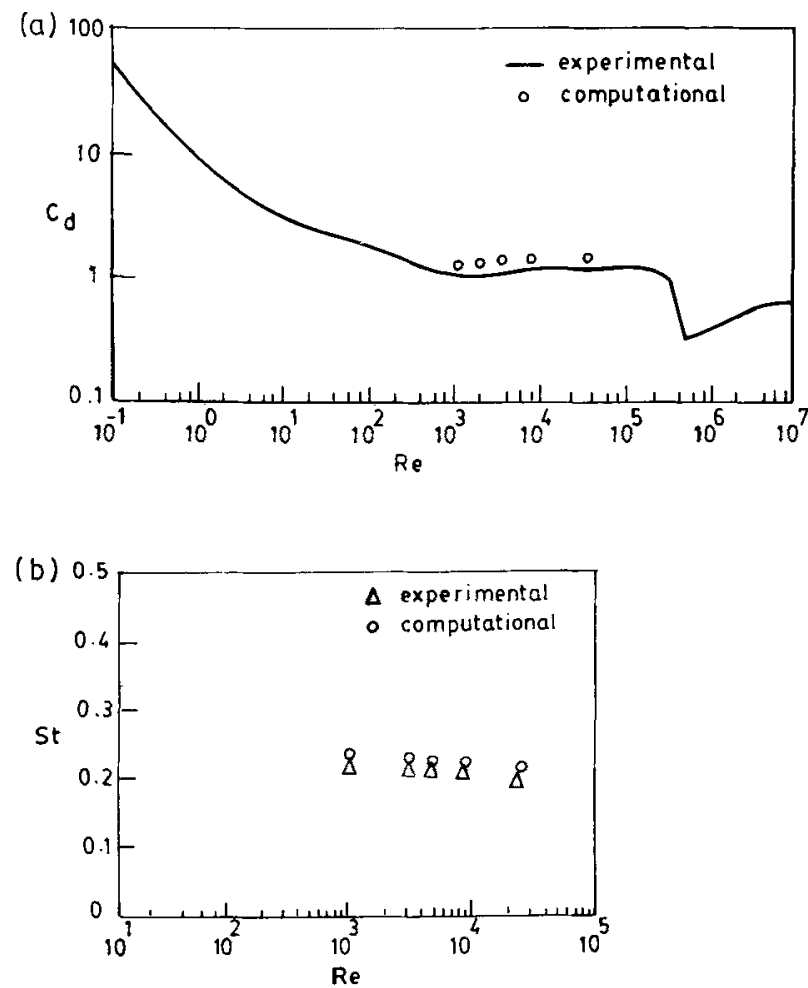

Figure 6. Instantaneous (c) vorticity contours, (d) time-deperdent drag and lift coefficients for a circular cylinder for $\operatorname{Re}=40,000$ at time $=45$.
Figure 7. Comparison between experimental and numerical results of (a) the drag coefficient, (b) the Stroubal number, at different values of Reynolds number. 
The authors wish to thank the referees for their valuable comments and suggestions which helped them to improve the presentation. One of the authors, Rao, wishes to express his gratitude to Professor M Kawahara of Chuo University. Tokyo, for useful suggestions.

\section{References}

Bouard R. Coutanceau M 1980 The early stage of development of the wake behind an impulsively started circular cylinder for $40<\mathrm{Re}<10^{4}$. J. Fluid Mech. 101: 583-607

Collins W M. Dennis S C R 1973 Flow past an impulsively started circular cylinder. J. Fluid Mech. 60: $105-127$

Coutanceau M. Bouard R 1977 Experimental determination of the main features of the viscous flow in the wake of a circular culiner in uniform translation. Part 2. Unsteady flow. J. Fhid Mech. 79: 257-272

Gerrard J H 1967 Numerical computation of the magnitude and frequency of the lift on a circular cylinder, Philos. Trams. R. Soc. 261: 137-162

Gerrad J H 1978 The wakes of cylindrical bluff bodies at low Reynolds number. Philos. Trans. R. Soc. 288: $351-382$

Hariow F H. Welch J E 1965 Numerical calculation of time-dependent viscous incompressible flow of fluid free-surface. Phis. Fluids 8: 2182- 2189

Honji H. Taneda S 1969 Unsteady flow past a circular cylinder. J. Phys. Soc. Jpn. 27: 1668-1677

Kawaguti $M 1953$ Numerical solution of the Navier-Stockes equations for the flow around a circular cylinder at Reynolds number 40. J. Phys. Soc. Jpn. 8: $747 . .757$

Kawaguti M. Jain P 1966 Numerical study of a viscous fluid flow past a circular cylinder. J. Phys. Soc. Jpn. 21. 2055-2062

Kawamura T. Kuwahara K 1984 Computation of high Reynolds number flow around a circular cylinder with surface roughness, AIAA-84-0340

Kawamura T. Kuwahara K 1985 Direct simulation of a turbulent inner flow by finite difference method, AIAA- $85-0376$

Kuwahara K 1978 Study of flow past a circular cylinder by an inviscid model. J. Phys. Soc. Jpn. 45: 292-297

Naumann A. Morsbach M, Kramer C 1966 The conditions of separation and vortex formation past cylinders. AGARD Conf. Proc. 4: 539-574

Patel V A 1976 Time dependent solutions of the viscous incompressible flow past a circular cylinder. Comput. Fluids 4: 13-25

Phuoc Loc T A. Bouard R 1985 Numerical solution of the early stage of the unsteady viscous flow around a circular cylinder: a comparison with experimental visualization and measurements. $J$. Fluid Mech. 160: $93-117$

Rao P M, Kuwahara K 1990 Finite difference simulation of time dependent flow around an oscillating cylinder. Accepted for the presentation 13th 1MACS. July 22-26, 1991, Dublin, Ireland

Rao P M. Kuwahara K. Shimizu M. Tsuboi K 1990a Numerical study of the unsteady flow around a transverscly and longitudinally oscillating cylinder in a uniform flow at high Reynolds numbers. $J$. Fluid Mech.

Rao P M, Kuwahara K. Tsuboi K 1990b Simulation of unsteady viscous flow around a longitudinally oscillating circular cylinder in a uniform flow. Appl. Math. Model. (submitted)

Rao P M, Kuwahara K, Tsuboi K 1990c A direct simulation of the flow around a circular cylinder sinusoidally oscillating at low Kculegan-Carpenter numbers. Accepted for the Presentation ICES 91 PATRAS, Apri] $21-26,1991$, Greece

Roshko A 1961 Experiments on the flow past a circular cylinder at very high Reynolds number. J. Fluid Mech. 10; 345-356

Sarpkaya T, Schoaff R L 1979 Inviscid model of two-dimensional vortex shedding by a circular cylinder. AIAA J. 17: 1193-1200

Schlichting H 1968 Boundary layer theory (New York: McGraw-Hill)

Takami H, Keller H B 1969 Steady two dimensional viscous flow of an incompressible fluid past a circular cylinder. Phys. Fluids, Stuppl. II 12:51-56

Taneda S 1977 Visual study of unsteady separated flows around bodies. Prog. Aerosp. Sci. 17: 287-348

Thom A 1933 The flow past circular cylinders at low speeds. Proc. R. Soc. London A141: 651-669

Wei T, Smith C R 1986 Secondary vortices in the wake of circular cylinders. J. Fluid Mech. 169: 513-533 